\title{
Electron-wall interaction in Hall thrusters ${ }^{\text {a) }}$
}

\author{
Y. Raitses ${ }^{\text {b) }}$ and D. Staack \\ Princeton Plasma Physics Laboratory, Princeton, New Jersey 08543 \\ M. Keidar \\ University of Michigan, Ann Arbor, Michigan 48109 \\ N. J. Fisch \\ Princeton Plasma Physics Laboratory, Princeton, New Jersey 08543
}

(Received 22 November 2004; accepted 22 February 2005; published online 2 May 2005)

\begin{abstract}
Electron-wall interaction effects in Hall thrusters are studied through measurements of the plasma response to variations of the thruster channel width and the discharge voltage. The discharge voltage threshold is shown to separate two thruster regimes. Below this threshold, the electron energy gain is constant in the acceleration region and therefore, secondary electron emission (SEE) from the channel walls is insufficient to enhance electron energy losses at the channel walls. Above this voltage threshold, the maximum electron temperature saturates. This result seemingly agrees with predictions of the temperature saturation, which recent Hall thruster models explain as a transition to space-charge saturated regime of the near-wall sheath. However, in the experiment, the maximum saturation temperature exceeds by almost three times the critical value estimated under the assumption of a Maxwellian electron energy distribution function. The channel narrowing, which should also enhance electron-wall collisions, causes unexpectedly larger changes of the plasma potential distribution than does the increase of the electron temperature with the discharge voltage. An enhanced anomalous crossed-field mobility (near wall or Bohm-type) is suggested by a hydrodynamic model as an explanation to the reduced electric field measured inside a narrow channel. We found, however, no experimental evidence of a coupling between the maximum electron temperature and the location of the accelerating voltage drop, which might have been expected due to the SEE-induced near-wall conductivity. (C) 2005 American Institute of Physics.
\end{abstract}

[DOI: $10.1063 / 1.1891747]$

\section{INTRODUCTION}

The Hall thruster ${ }^{1,2}$ (HT) is a spacecraft propulsion device, which generates the thrust due to electrostatic acceleration of ions in quasineutral plasma. The plasma discharge is sustained in the axial electric and radial magnetic fields applied in an annular channel. Because of the reduced electron mobility across the magnetic field, a substantial axial electric field can be maintained in quasineutral plasma and the electrons can effectively ionize the neutral gas atoms. Under such conditions, the electric field supplies energy mainly to accelerate the unmagnetized ions. Part of the energy goes also to heat the electrons, which diffuse across the magnetic field and dissipate the gained energy mainly on ionization of neutral atoms and collisions with the channel walls. The electron crossed-field current is necessary to sustain the thruster discharge, but the power it carries away from the accelerating region results in reduction of the thruster efficiency, ${ }^{2,3}$

Existing HTs operate in a subkilovolt discharge voltage range using xenon gas. In the input power range of 0.2-50 $\mathrm{kW}$ the thruster efficiency is $40 \%-60 \%{ }^{4,5}$ The xenon gas flow is typically almost completely ionized. ${ }^{1,2}$ The plasma is weakly collisional. The electron temperature $T_{e}$ is $20-50 \mathrm{eV}$.

\footnotetext{
a) Paper JI1B 2, Bull. Am. Phys. Soc. 49, 206 (2004).

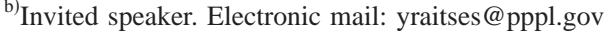

Under the assumption of a Maxwellian electron energy distribution function $(\mathrm{EDF})$, the electron temperature of this range is sufficiently large to induce a strong secondary electron emission (SEE) from ceramic channel walls. ${ }^{6}$ A strong SEE may enhance electron-wall collisions leading to additional power losses. ${ }^{1,7-10}$

It is well known since the classical work of Hobbs and Wesson ${ }^{11}$ that the electrons emitted from a surface of the floating wall to the plasma reduce the potential drop in the near-wall sheath. ${ }^{11-13}$ The increased electron flux to the wall, $\Gamma_{\mathrm{e}}$, is balanced by the flux of ions, $\Gamma_{\mathrm{i}}$, and secondary electrons, $\Gamma_{\mathrm{es}}: \Gamma_{e}=(1-\delta)^{-1} \Gamma_{i}$. When the SEE coefficient, $\delta$ $\equiv \Gamma_{\mathrm{es}} / \Gamma_{\mathrm{e}}$, reaches approximately 1 the sheath becomes space-charge saturated (SCS). In the SCS regime the wall acts as an extremely effective particle and energy sink. ${ }^{11,14}$

A number of recent theoretical studies suggested the occurrence of the SCS regime in HTs. ${ }^{8-10}$ According to these studies a saturation of the maximum electron temperature is caused due to enhanced electron energy losses on the walls. In addition to electron cooling, it is also believed that the SEE from the channel walls can enhance the electron crossed-field diffusion (so-called near-wall conductivity ${ }^{1}$ ). Barral et al. ${ }^{9}$ predicted a dominant role of the near-wall conductivity in the SCS regime. This prediction seems to correlate with the measurements of voltage versus current $(V-I)$ characteristics $^{15}$ of the thruster discharge. The $V-I$ character- 
istics exhibit a typical jump of the discharge current above a certain discharge voltage, which is different for different channel wall materials with different SEE properties. ${ }^{15,16}$ However, kinetic simulations ${ }^{17-20}$ suggest that in a weakly collisional thruster plasma the electron EDF is depleted at high energies due to wall losses. A similar depletion effect of wall losses on electron EDF is also known in other types of low-pressure gas discharges. ${ }^{21}$ For HTs, because of a small SEE, a minor contribution of electron-wall collisions is expected to the electron transport. ${ }^{17,19,20}$

According to a conventional model of electron-wall interaction, ${ }^{7-10,17}$ the frequency of electron-wall collisions depends on the electron flux and the channel geometry, $v_{e w}$ $\propto \Gamma_{e} / n_{e} h$, where $h$ is the channel width and $n_{e}$ is the electron density. Apart from the SEE, variations of the channel width should directly affect particle and electron energy losses on the walls and, if the SEE happens to be sufficiently strong, the near-wall conductivity. The present work will examine the macroscopic picture of how the plasma responds to variations of the electron-wall interaction with the channel width.

The influence of the channel wall material on the plasma flow and the thruster discharge characteristics were studied elsewhere. ${ }^{7,9,15,16}$ The ability to control the plasma flow through a segmentation of the thruster channel has been demonstrated both theoretically ${ }^{3,22}$ and experimentally. ${ }^{22,23}$ In previous works the channel width effects were mostly considered and studied with respect to ionization efficiency ${ }^{24}$ and thruster scaling. ${ }^{1,2}$ It is interesting to use the channel width variations along with measurements of plasma parameters as a research tool in order to validate the existing theories of electron-wall interaction effects such as the near-wall conductivity.

This paper is organized as follows. In Sec. II we describe the experimental setup and the experimental procedure. Experimental results are presented and discussed in Sec. III. Section IV compares these results with simulations. A few remarks on the thruster performance are given in Sec. V. Conclusions are summarized in Sec. VI.

\section{EXPERIMENTAL SETUP}

\section{A. Thruster configurations}

A $2 \mathrm{~kW}$ laboratory Hall thruster ${ }^{25-27}$ consists of magnetic circuit, coaxial channel, the anode, which is also a gas distributor, and the hollow cathode neutralizer. A set of electromagnet coils produces the magnetic field in the channel. The magnetic field distribution in the channel is shaped by the magnetic core. In these experiments, the magnetic field is the same for all operating regimes and the channel configurations. Figure 1 shows the channel and simulation results of the magnetic field for the used experimental conditions.

The thruster channel is made from a grade HP boron nitride $(\mathrm{BN})$ ceramic material. Under the assumption of a Maxwellian electron EDF the SEE yield from this material approaches $\sim 1$ when $T_{e}$ of primary electrons is equal to $\approx 18 \mathrm{eV} \cdot{ }^{6,19,28}$ The effective channel length taken from the anode to the channel exit is $46 \mathrm{~mm}$. The channel width is measured between the inner and outer channel walls. In one thruster geometry, the channel has the outer diameter of 123

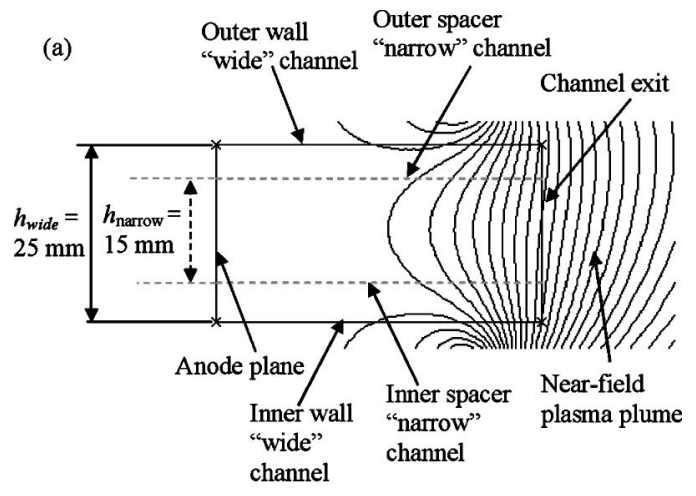

(b)

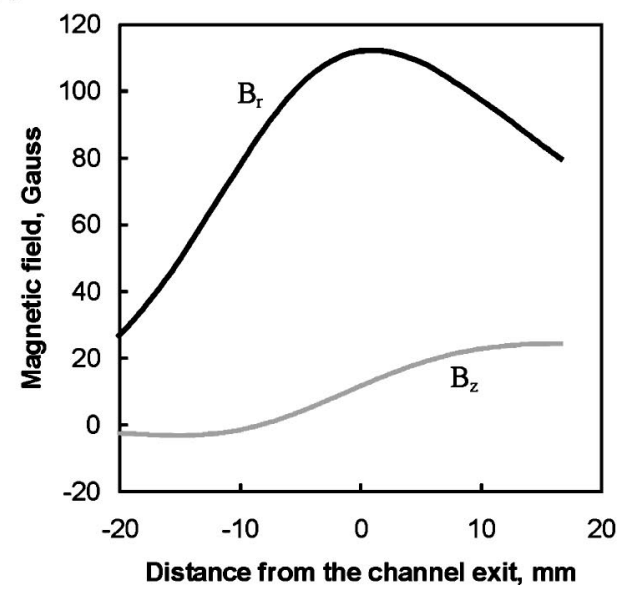

FIG. 1. Schematic of the thruster channel with superimposed magnetic field lines (a). Magnetic field distribution along the channel median (b). The channel length is $46 \mathrm{~mm}$. The magnetic field distribution was simulated for the experimental conditions.

$\mathrm{mm}$ and the width of $25 \mathrm{~mm}$. We call this thruster configuration as "wide." In the second thruster configuration, referred as "narrow," two boron nitride spacers are added to the inner and outer channel walls of the wide channel. With each spacer of $5 \mathrm{~mm}$ thick, the width of this channel is 15 $\mathrm{mm}$.

The magnetic field at the inner wall of the narrow channel is 170 gauss, while for the wide channel 240 gauss. The maximum magnetic field along the channel median is 113 gauss (Fig. 1). Therefore, when we refer to the channel narrowing it includes also the reduction of the mirror near the inner wall. The mirror ratios are $\approx 2.1$ and $\approx 1.5$ for the wide and narrow channel configurations, respectively. The radial magnetic field near the outer wall with and without spacer is $\sim 100$ gauss. For the narrow channel, one may expect a degradation of the plasma confinement because of the reduced mirror ratio. As discussed previously, we expect the channel narrowing to enhance plasma-wall interaction as well.

\section{B. Diagnostics}

We use and operate floating movable probe in hot (emissive) and cold regimes and then obtain the plasma potential and the electron temperature. The probe design and setup are described in detail elsewhere. ${ }^{26}$ Briefly, the probe filament is 


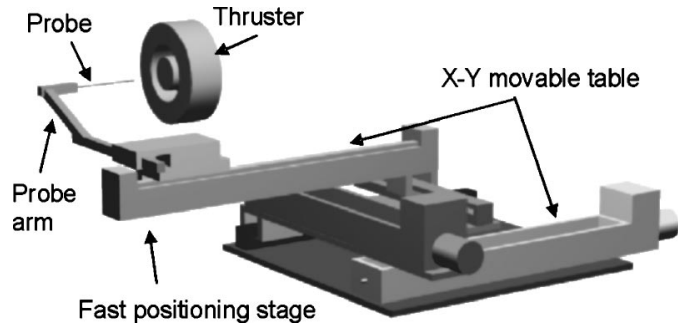

FIG. 2. Movable probe setup.

constructed of $0.1 \mathrm{~mm}$ thoriated tungsten wire. The probe is introduced into the thruster along the channel median by a fast positioning stage as shown in Fig. 2. The probe has a segmented shield in order to reduce plasma perturbations, which can be induced from the probe tube made from high SEE alumina ceramic. For the results presented in this paper, probe-induced changes of the discharge current were $\leqslant 15 \%$. The spatial resolution of the emissive probe in the axial direction is approximately half the probe filament length $(<2.5 \mathrm{~mm})$.

A $25.4 \mathrm{~mm}$ diameter flat electrostatic probe with a guarding sleeve measures the total ion flux for all ions coming from the thruster. The plume probe is mounted on a rotational positioning stage. The probe axis is pointed to the thruster center at the exit plane. The radius of probe rotation around the thruster center is $730 \mathrm{~mm}$. Both probe and sleeve are biased $-30 \mathrm{~V}$ with respect to ground.

The thruster experiments take place in a $28 \mathrm{~m}^{3}$ vacuum vessel equipped with two cryogenic pumps. ${ }^{25-27}$ We operated the thruster at a constant xenon flow rate of 19 SCCM (SCCM-cubic centimeter per minute at STP) in the discharge voltage range of $150-600 \mathrm{~V}$. The background pressure did not exceed $6 \mu$ torr. The thruster electrodes were floating with respect to ground.

\section{Experimental procedure}

For each channel configuration, the fast probe and plume probe measurements were performed in separate sets of experiments. In each operating point, the thruster was allowed to reach a steady state operation, which was monitored by the discharge current. It could take more than half an hour, in particular, for operation at high discharge voltages, to reach a steady state. Such a long transitional regime, which is characterized by large values of the discharge current, was early reported by Hofer and Jankovsky ${ }^{29}$ for a different HT. After the discharge current reduces and reliably saturates, a floating potential of the fast probe is measured first without the heating and then with heating on. The measured data from multiple probe insertions are processed as described in detail in Ref. 26.

For the determination of the plasma parameters, we assume the plasma electrons to be Maxwellian and take into account the effect of space-charge limited emission from the emissive probe on the floating potential. ${ }^{12}$ For xenon plasma an approximate relation between the plasma potential $\phi_{\mathrm{pl}}$ and the floating potential of the emissive probe $\phi_{\mathrm{fl}}^{\mathrm{em}}$ is $\phi_{\mathrm{pl}}=\phi_{f}^{\mathrm{em}}$ $+1.5 T_{e}$. For a cold probe, we use the classical expressions of the floating potential $\phi_{f}^{\mathrm{cl}}$, which for xenon plasma is $\phi_{f}^{\mathrm{cl}}$ $=\phi_{\mathrm{pl}}-5.77 T_{e}$, where $T_{e}$ is the electron temperature. From these equations, the electron temperature is

$$
T_{e}=\frac{\phi_{f}^{\mathrm{em}}-\phi_{f}^{\mathrm{cl}}}{4.27} \text {. }
$$

The electric field is obtained by numerical differentiation of the plasma potential distribution.

The assumptions of the Maxwellian EDF and the planar sheath between the probe wire and the plasma may introduce uncertainties in determination of the electron temperature [Eq. (1)]. We placed a biased planar probe $d=0.74 \mathrm{~mm}$ $>\lambda_{D}$ for typical HTs near the thruster exit to obtain the electron temperature from the probe $V-I$ characteristics. Discrepancies between the fast probe and the biased probe were $<10 \%$ in discharge voltage range of $200-300 \mathrm{~V}$. It is within the reproducibility error of the fast probe measurements ( $<15 \%$ ). Different probe techniques used in Hall thruster studies elsewhere ${ }^{30-33}$ give also comparable results and profiles. It is believed therefore that the electron temperature defined by Eq. (1) gives an approximate estimate of the electron mean energy.

The total ion flux from the thruster is obtained by integrating over the measured ion flux angular distribution. ${ }^{23} \mathrm{~A}$ standard deviation of these measurements is less than $3 \%-$ $5 \%$. Using the discharge current and flow rate measurements, we deduce the current utilization, $\eta_{C} \equiv I_{i} / I_{d}$, which characterizes how effectively the magnetic field impedes the axial electron current, and the propellant utilization, $\eta_{P} \equiv I_{i} / I_{\dot{m}}$, which characterizes the ionization efficiency. ${ }^{2}$ Here, $I_{\dot{m}}$ $=e \dot{m} / M_{\mathrm{Xe}}$ is the supplied flow rate in current unit, $\dot{m}$ is the mass flow rate, $M_{\mathrm{Xe}}$ is the xenon atom mass and $e$ is the electron charge.

\section{EXPERIMENTAL RESULTS AND DISCUSSIONS}

\section{A. Discharge characteristics}

Figure 3 shows the $V-I$ characteristics and the current utilization. For the wide channel configuration, $\approx 90 \%$ of the supplied gas flow rate is ionized when the discharge voltage increases above $200 \mathrm{~V}$. For the narrow configuration, the shape of the $V-I$ characteristics is affected by changes of the ionization efficiency and the current utilization. The ion current saturates at $75 \%$ of the supplied gas flow rate, at $V_{d}$ $\sim 300-400 \mathrm{~V}$, but then increases to about $95 \%$ at $500 \mathrm{~V}$.

\section{B. Plasma potential and electron temperature}

The most striking effect of the channel narrowing (and the mirror reduction) is on the axial distributions of the plasma parameters. Figure 4 and 5 exemplify this effect for $V_{d}=250 \mathrm{~V}$. A significant voltage potential drop occurs in the acceleration region with a strong radial magnetic field. By narrowing the channel, we push the acceleration region to the near-field plasma plume. The local maximum of the electric field and the local maximum of the electron temperature are also shifted outward (Fig. 5).

Figure 6 summarizes this effect for different discharge voltages. For comparison, we show the ratio of the outside 


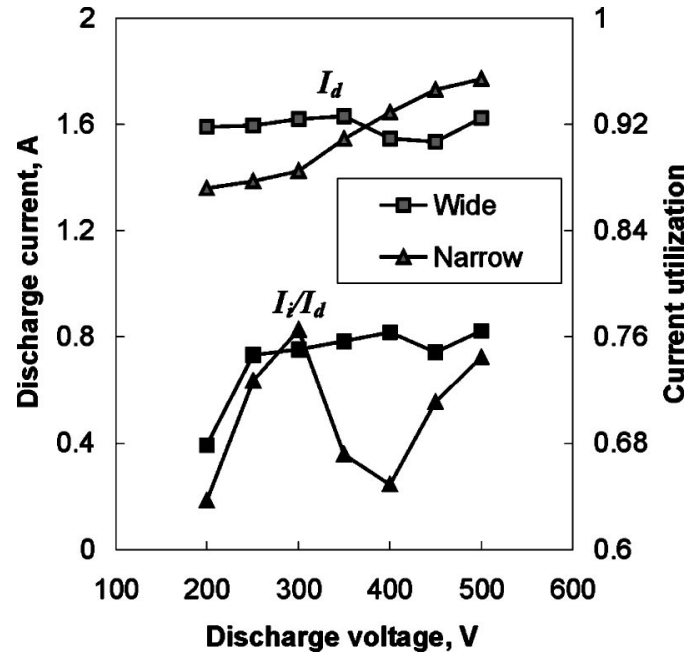

FIG. 3. Voltage vs current characteristics of the thruster discharge $I_{d}$ and the current utilization $I_{i} / I_{d}$ measured for xenon gas flow of 19 SCCM and two thruster configurations with two different channel widths: $25 \mathrm{~mm}$ (wide) and $15 \mathrm{~mm}$ (narrow). Magnetic field is not changed.

voltage drop measured between the channel exit and the cathode plane, $V_{\text {out }} \equiv \phi_{\text {exit }}-\phi_{\text {cath }}$, to the discharge voltage for two thruster configurations. The cathode plane is determined from the electric field distribution as shown in Fig. 5(b). Thus, we exclude our estimations from relatively smooth potential variations in the expanding plasma. With the discharge voltage the same, the voltage drop ratio for the narrow channel configuration is generally greater than that in the wide channel case.

\section{Maximum electron temperature}

The maximum electron temperature for different discharge voltage is shown in Fig. 7. In each thruster configuration there is roughly the same discharge voltage threshold

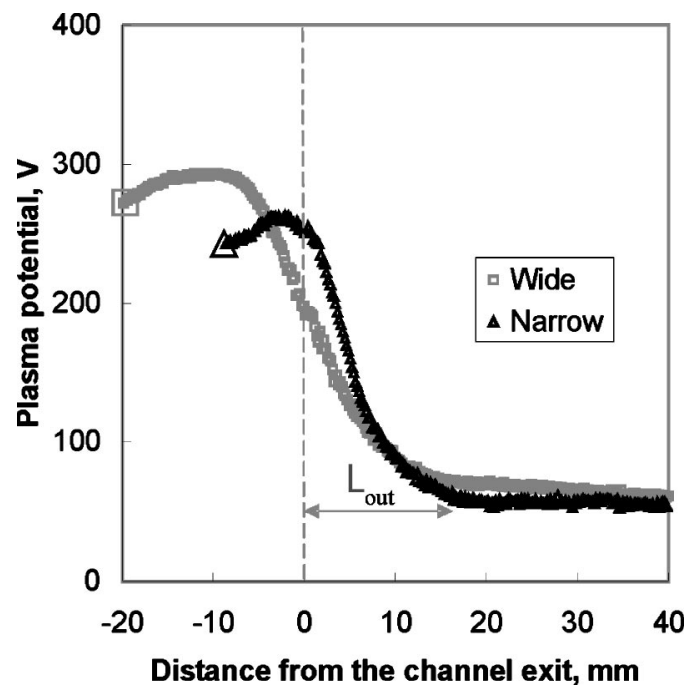

FIG. 4. Effect of the channel width on the plasma potential distribution. Plasma potential distribution is along the channel median of the narrow (15 $\mathrm{mm}$ width) and wide (25 $\mathrm{mm}$ width) channels measured for the discharge voltage of $250 \mathrm{~V}$. The anode placement is $-46 \mathrm{~mm}$. $L_{\text {out }}$ is the length of the plume part of the acceleration region outside the channel exit.

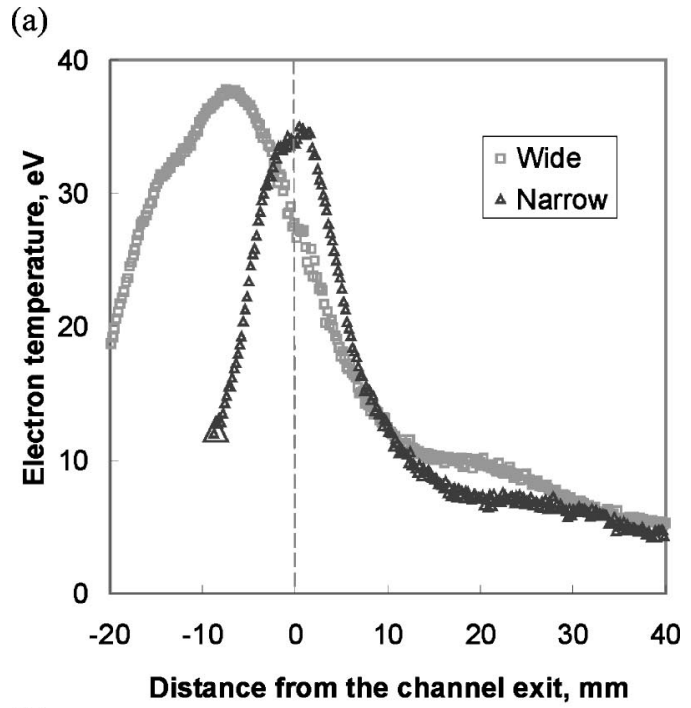

(b)

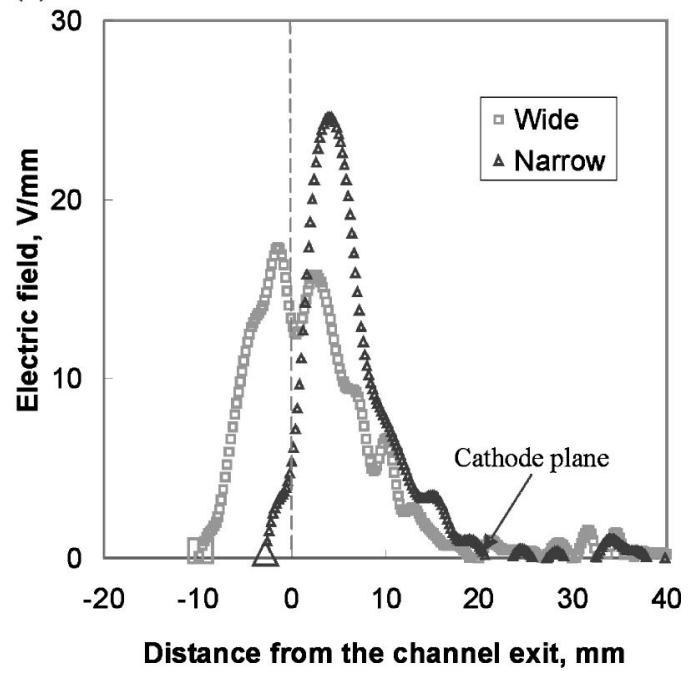

FIG. 5. Effect of the channel width on the electron temperature (a) and the electric field (b). The anode placement is $-46 \mathrm{~mm}$. The discharge voltage is $250 \mathrm{~V}$.

$(\sim 400 \mathrm{~V})$, which separates two temperature regimes. Below the voltage threshold, the maximum electron temperature increases nearly linear with the discharge voltage. Above the voltage threshold, the maximum temperature saturates. Despite this difference, in each operating point there is a part of the acceleration region where the local electron temperature increases almost linearly with the local plasma potential (Fig. 8). A linear relationship between the local plasma parameters yields a constant electron energy gain, i.e., $\nabla T_{e}=\beta e \boldsymbol{\phi}$, where $\beta \approx$ const. For the wide channel, this relationship is seen both inside the channel and in the near field plasma plume (Fig. 8). In our previous study ${ }^{34}$ a similar behavior was observed and attributed to minor SEE effects on electron energy losses to the walls. These results suggest that a minor role of the SEE appears above the voltage threshold as long as the electron energy gain continues to be constant.

It is worth mentioning that in other measurements taken without a long waiting time, presumably during a transitional regime, the discharge current is typically larger than in steady state operation. For this transitional regime, we ob- 


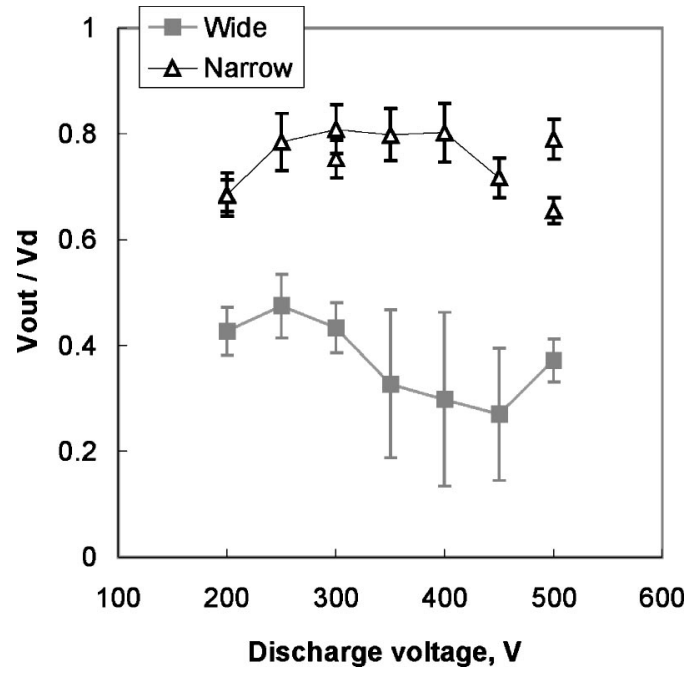

FIG. 6. Effect of the channel width on the acceleration region: the outside voltage drop in the near-field plasma plume as a function of the discharge voltage.

served very similar dependence of the maximum electron temperature on the discharge voltage with approximately the same voltage threshold as for a steady state operation. However, above the voltage threshold, the location of the maximum electron temperature is in the near field plasma plume. The results for this transitional regime will be discussed in a separate paper.

For the narrow channel configuration, the acceleration region is almost completely reduced to being located in the near field plasma plume (Figs. 5 and 6). A linear relationship between the local electron temperature and plasma potential are also seen in this region (Fig. 8). The plasma-wall interaction occurs probably near the channel exit where the electrons bounce along magnetic field lines intersecting the sidewalls of the ceramic channel (Fig. 1). The ionization takes



FIG. 7. The dependence of the maximum electron temperature on the discharge voltage for two thruster configurations. For the wide channel configuration, the electron temperature reaches its maximum inside the channel while for the narrow channel configuration it does outside the channel (See Fig. 5).

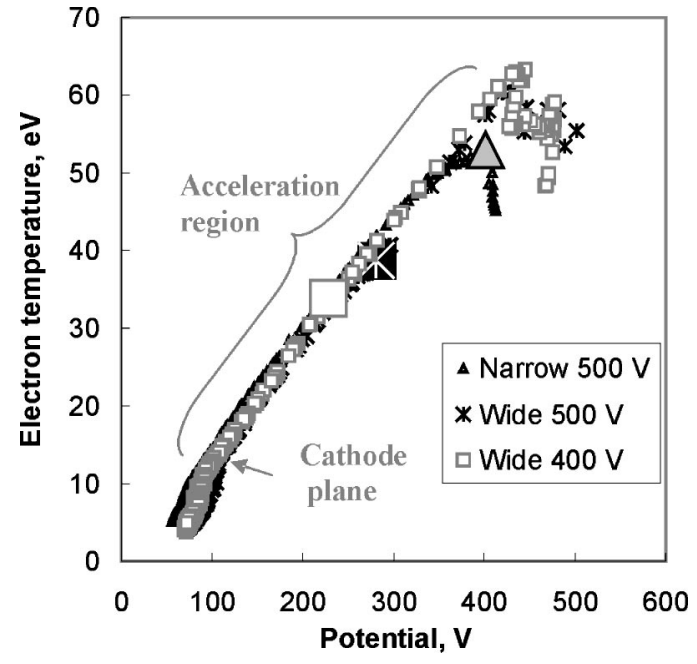

FIG. 8. Variations of the local electron temperature with the local plasma potential for the narrow channel $\left(V_{d}=500 \mathrm{~V}\right)$ and the wide channel $\left(V_{d}\right.$ $=400 \mathrm{~V}$ and $500 \mathrm{~V}$ ) configurations. For each regime and configuration, the enlarged marker corresponds to the local parameters at the channel exit. Notations explain approximate regions where measurements were taken for the wide channel configuration. For the wide channel cases, the local maximum of the electron temperature is $3-6 \mathrm{~mm}$ upstream of the channel exit. A constant electron energy gain is seen in the acceleration region of each configuration.

place in the vicinity of the channel exit, but probably more intense ionization occurs inside the channel (larger neutral density than in the plume), where the electron temperature is seen to drop rapidly. The saturation temperature is roughly three times higher than the critical value $(\sim 18 \mathrm{eV})$ estimated for Maxwellian electron EDF. ${ }^{28}$ This result may additionally support predictions of kinetic studies ${ }^{17-19}$ of a reduced role of the SEE, because of an electron EDF depleted at high energies.

\section{Discussions of discharge voltage effects}

For the narrow channel configuration, the electric field outside the channel exit is larger than that it is inside the channel. This holds both below and above the voltage threshold. The current utilization is almost the same except for some differences for $300 \mathrm{~V}<V_{d}<500 \mathrm{~V}$. The location of the voltage drop depends on the axial variation of the electron mobility. ${ }^{35}$ It is difficult to quantify changes of the electron mobility inside the channel, because if the electron EDF departs from Maxwellian due to wall losses, it may depart differently at different discharge voltages. However, it is still informative to evaluate the average electron mobility across the magnetic field in the plume part of the acceleration region, which is located outside the channel exit. in the nearfield plasma plume. For that purpose, we consider two operating points of the thruster with the narrow channel: $300 \mathrm{~V}$ and $500 \mathrm{~V}$, and use Ohm's law to determine the average electron mobility $\mu_{\perp}, v_{e z}=\mu_{\perp}\left[E-\left(1 / e n_{e}\right) d\left(n_{e} T_{e}\right) / d z\right]$. Here $v_{e z}$ is the electron velocity in the axial direction $z, E$ is the electric field, $e$ is the electron charge and $n_{e}$ is the electron density. Neglecting for now the electron pressure gradient, the average mobility in the near-field plasma plume can be expressed as $\bar{\mu}_{\perp} \approx E_{\text {out }} / v_{e z}$, where a $E_{\text {out }} \approx V_{\text {out }} / L_{\text {out }}$, is the 
average electric field in the near-field plasma plume and $L_{\text {out }}$ is length of this region measured between the channel exit and the cathode plane. Within accuracy of our measurements, we take $V_{\text {out }} \approx 230 \mathrm{~V}$ and $L_{\text {out }} \approx 20 \mathrm{~mm}$ for $300 \mathrm{~V}$ and $V_{\text {out }}$ $\approx 360 \mathrm{~V}$ and $L_{\text {out }} \approx 27 \mathrm{~mm}$ for $500 \mathrm{~V}$ (Fig. 6). In the acceleration region, we may assume a monoenergetic ion flow with no particle sources and sinks, $\Gamma_{\text {ion }}(z) \approx I_{i} / e A \approx$ const, where $A$ is the cross-sectional area of the plasma in the nearfield plasma plume. From the measured angular ion flux distribution, we found that in the narrow channel case the plasma plume angle is approximately the same $\sim \approx 110^{\circ}$ for $300 \mathrm{~V}$ and $500 \mathrm{~V}$. It may justify the assumption of equal cross-sectional areas of the plasma in the plume part of the acceleration region. Applying a plasma quasineutrality, the axial electron velocity can be described with a simplified expression similar to that of Ref. 36, $v_{e z}(z) \approx\left(\eta_{c}^{-1}-1\right) V_{\text {ion }}(z)$, where $V_{\text {ion }}(z) \approx \sqrt{2 e[\Delta \phi(z)] / M_{\mathrm{Xe}}}$ is the ion velocity. Since we use the average electric field (constant), we assume $\Delta \phi \approx V_{\text {out }}$. The current utilization for 300 $\mathrm{V}$ and $500 \mathrm{~V}$ is $\approx 0.75$ (Fig. 3). Hence, the electron mobility ratio $\mu_{\perp}^{300 \mathrm{~V}} / \mu_{\perp}^{500 \mathrm{~V}} \approx\left(\sqrt{V_{\text {out }}} / L_{\text {out }}\right)_{300} /\left(\sqrt{V_{\text {out }}} / L_{\text {out }}\right)_{500}$ is equal roughly to 1 . Note that using the assumption $\Gamma_{\text {ion }}(z) \approx$ const, we can deduce a plasma density distribution and then estimate the electron pressure. By including the average electron pressure gradient (through Ohm's law), we obtained about $30 \%$ reduction of the mobility ratio.

Because the average electron mobility and a fraction of the voltage drop outside the channel exit change little with the discharge voltage, we deduce that changes of the average electron mobility inside the channel are also insignificant. In that case, if the observed temperature saturation is a manifestation of strong SEE effects, then the question remains how to explain large electron mobility inside the channel at lower electron temperatures obtained below the discharge voltage threshold. In the other words, there is no clear experimental evidence of direct coupling between the electron mean energy and the average electron mobility, which one could expect due to the SEE-induced near-wall conductivity.

Hagelaar et $a l^{35}$ considered parametrically a situation when the near-wall conductivity reduces five times as the discharge voltage changes from $600 \mathrm{~V}$ to $300 \mathrm{~V}$ (cases 2 and 3 of Ref. 35). The near-wall conductivity inside the channel and the Bohm-type mobility outside the channel exit were assumed and controlled by empirical coefficients. The mobility coefficients for near-field plume region were equal in both voltage cases. Simulated results ${ }^{35}$ suggest that under such conditions a fraction of the discharge voltage, which drops outside the channel at $600 \mathrm{~V}$, is approximately twice larger than that for $300 \mathrm{~V}$. In the experiment, the situation is different. The increase of the discharge voltage does not lead to the increase of the outside voltage drop because of the crossed-field mobility does not increase within the studied discharge voltage range.

Finally, an insignificant reduction of the voltage ratio obtained between 300 and $500 \mathrm{~V}$ correlates with an increase of the ionization efficiency (from Fig. 3) and, therefore, may be explained as a reduction of the electron mobility inside the channel because of rarer electron-atom collisions inside the channel. As it is explained in the following section, clas- sical electron transport is not an adequate model to explain the experimental discharge current in the discharge voltage range used in these experiments.

\section{HALL THRUSTER MODELLING AND COMPARISON WITH EXPERIMENT}

In this section, we attempt to simulate the channel narrowing effect on the plasma flow. For the thruster model, we consider the plasma flow, which starts in the near anode region, and has the lateral boundaries near the dielectric wall. Quasineutrality is assumed. The plasma presheath-sheath interface is considered as the lateral boundary for the plasma flow region. This model was described in details elsewhere. ${ }^{7,22}$ Let us briefly review the main points of the model and some of its peculiarities.

We employ a hydrodynamic model in two-dimensional (2D) domain assuming that the system reaches a steady state. Only a radial component of the magnetic field is considered. The momentum and mass conservation equations for electrons, ions, and neutrals under such conditions have the following form:

$$
\begin{aligned}
& n m_{i}\left(\boldsymbol{V}_{i} \boldsymbol{\nabla}\right) \boldsymbol{V}_{i}=n e \boldsymbol{E}-\boldsymbol{\nabla} P_{i}-\beta_{i} n m_{i} n_{a}\left(\boldsymbol{V}_{i}-\boldsymbol{V}_{a}\right), \\
& \boldsymbol{\nabla}\left(V_{i} n\right)=\beta_{i} n n_{a}, \\
& \boldsymbol{\nabla}\left(\boldsymbol{V}_{a} \boldsymbol{n}_{a}\right)=-\beta_{i} n n_{a}, \\
& 0=-e n(\boldsymbol{E}+\boldsymbol{V} \times \boldsymbol{B})-\boldsymbol{\nabla} P_{e}-n \nu_{e f} m_{e}\left(V_{e}-V_{i}\right),
\end{aligned}
$$

where $n$ is the plasma density, $\beta_{i}$ is the ionization rate, $n_{a}$ is the neutral density, $V$ is the velocity, and $\nu_{\text {ef }}$ is the effective collision frequency. The subscripts $e, i$, and $a$ denote electron, ion, and neutral atom, respectively.

Further model simplification includes consideration of the one-dimensional flow of the neutral gas atoms. Finally, electron energy equation and electron transport are considered in a one-dimensional framework along the channel median. Since only the radial magnetic field component is considered, the electron transport is much greater in the azimuthal direction $(\mathbf{E} \times \mathbf{B}$ drift $)$ than in the axial direction (drift diffusion due to collisions). Therefore, in most HT models, except some special cases ${ }^{37}$ an assumption of a constant "thermalized" potential ${ }^{1}$ along the magnetic field is used. This assumption reflects the fact that electrons can freely move along magnetic field line and therefore potential of the electric field is constant along magnetic field line (with accuracy of the electron pressure gradient). If the electron temperature is constant along each magnetic field line, then

$$
\varphi-\frac{k T_{e}}{e} \ln n=\text { const. }
$$

Using this equation it is possible to reduce the twodimensional electron transport and electric field calculation to a one-dimensional problem.

The electron temperature is calculated along the channel median as a balance between the Joule heating, ionization, and wall losses as described elsewhere. ${ }^{7,22}$ The channel is made from dielectric (ceramic) walls and therefore plasma- 
wall interaction must be considered. The sheath region in front of the dielectric surface is considered as collisionless. We use an effective coefficient of the SEE, which is assumed to be a linear function of the electron temperature up to $\delta$ $=0.98{ }^{6}$

One of the important effects to be considered with respect to the channel width variation is the anomalous electron transport across the magnetic field. It is well known that just the classical collision mechanism cannot explain the observed current in the Hall thruster. ${ }^{1,38}$ Electron mobility was found to be significantly larger than the typical values given by the classical mechanism most likely due to plasma turbulence ${ }^{19,38,39}$ and wall effects. ${ }^{1,9,35}$ Since detailed understanding of these anomalous transport mechanisms is lacking, we treat this problem using a semiempirical approach.

We consider that electron transport across the magnetic field is due to several collision mechanisms: electron-neutral collisions, electron-wall collisions, and anomalous (Bohm) diffusion: $\nu_{\mathrm{ef}}=\nu_{e n}+\nu_{w}+\nu_{B}$, where $\nu_{\mathrm{ef}}$ is the effective electron collision frequency. The anomalous electron collision frequency due to electric field fluctuation can be written in the following form:

$$
\nu_{B}=\frac{1}{\alpha 16} \omega_{c e},
$$

where $\alpha$ is the constant empirical parameter and $\omega_{c e}$ is the electron gyrofrequency.

The electron-wall collision frequency is difficult to describe because our experimental results suggest a minor SEE in regimes below the voltage threshold. With a low SEE, a commonly accepted model of electron-wall interaction gives a little contribution to the electron transport. However, in reality, the mechanism of electron-wall interaction is much more complicated; one should take into account many factors, such as surface roughness, ${ }^{1}$ non-Maxwellian electron distribution function, various electron scattering processes, ${ }^{40}$ etc. All these effects may contribute to the electron-wall interaction, but there is no detailed understanding of their mechanisms. Therefore, we adopt here a semiempirical approach to approach of Hagelaar et al. ${ }^{35}$ (however, keeping the evident dependence of the near-wall collision frequency on the channel width) describing the near-wall conductivity in the following way:

$$
\nu_{w}=\frac{1}{h} \beta 10^{7},
$$

where $h$ is the channel width in centimeters and $\beta$ is the constant empirical parameter.

The boundary conditions and numerical analysis are similar to those developed previously elsewhere. ${ }^{7,22,41} \mathrm{We}$ use the implicit two-layer method to solve the system of Eqs. (2)-(5). These equations are approximated by a two-layer, six-point scheme. An iterative self-consistent procedure for finding the plasma density, velocity, electron temperature, and potential distribution is employed similar to Ref. 41.

Figure 9 compares the experimental and simulated electron temperature distributions along the channel median in the case of wide channel. The electron temperature peaks

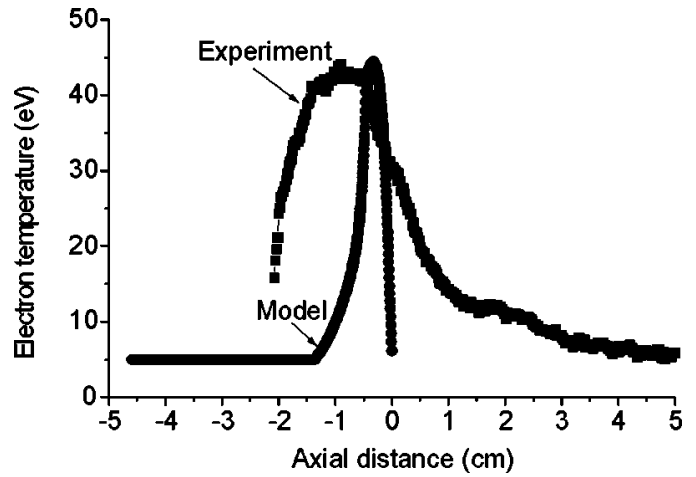

FIG. 9. Electron temperature distribution inside the wide channel. Comparison of simulations with experiment for $V_{d}=300 \mathrm{~V}$.

near the thruster exit plane where the electric field is highest. Then, the electron temperature decreases because of wall losses and ionization losses.

The electric field in the HT channel tends to concentrate itself in the region with the lowest mobility. For our thruster the results of simulations are shown in Fig. 10. The potential distribution along the channel median inside the channel is shown for different Bohm mobility coefficients. These results were obtained assuming only Bohm anomalous mobility inside the channel. Note that lower coefficient $\alpha$ [Eq. (7)] corresponds to higher electron conductivity across the magnetic field. Thus, the electron conductivity enhancement inside the channel can cause qualitatively similar changes of the plasma potential distribution as those obtained in the experiments (Figs. 4 and 6).

While it is not clear how channel width affects plasma turbulence and associated with this anomalous electron transport, it is expected that the channel width can affect electronwall collisions. Figure 11 illustrates this effect for two thruster configurations used in these experiments. It appears that only variations of the channel width are insufficient to reproduce the experimental result. Therefore one should take into account also change of the coefficient $\beta$. This reflects the fact that near-wall conductivity depends on the electron EDF, SEE, etc. In these experiments and simulations, we keep the same axial distribution of the magnetic field for both channel cases. However, the reduced magnetic mirror

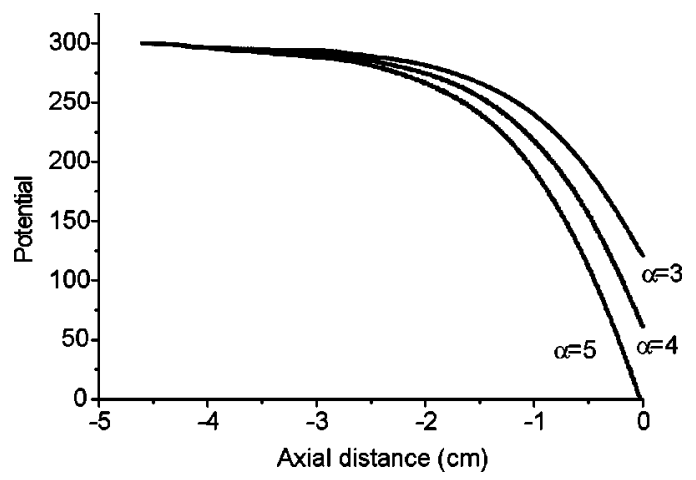

FIG. 10. Simulation results: Potential distribution inside the Hall thruster channel. Bohm anomalous conductivity effect. $\alpha$ is the empirical coefficient in Eq. (7). 




FIG. 11. Simulation results: Potential distribution inside the thruster channel with near-wall conductivity empirical coefficient as a parameter. $V_{d}$ $=300 \mathrm{~V}$. Electron anomalous transport is based on the near-wall effect. $\beta$ is the empirical coefficient in Eq. (8).

near the inner wall (Fig. 1) of the narrow channel might lead to larger electron energy losses and somehow contribute to a larger effect on the plasma potential distribution than the channel narrowing. The magnetic mirror near the inner wall may also affect electron $\mathrm{EDF}^{20}$ and therefore also the electron-wall interaction, leading to increased electron-wall collisions. A detail study of these effects is necessary, but it is out of scope of this paper. We mention a number of studies, which demonstrated the effect of the magnetic field topology on ionization efficiency and beam focusing properties in HTs. $1,42-44$

\section{A FEW REMARKS ON THRUSTER PERFOMANCE}

Having a larger potential drop in the near-field plasma plume may have some advantages and disadvantages for thruster applications. A possible advantage is that less energetic ions are able to strike the walls and cause the channel erosion. ${ }^{44}$ However, the ion acceleration occurs in the fringing magnetic field where the plasma flow is subjected to divergence because of defocusing equipotential surfaces ( $\mathbf{E}$ $\left.=-\mathbf{v}_{e} \times \mathbf{B}\right){ }^{44,45}$ The plasma divergence is usually measured in terms of a half plasma plume angle for $90 \%$ or $95 \%$ of the total ion flux coming from the thruster. In our previous studies, ${ }^{22,23}$ we demonstrated that using low SEE segmented electrodes placed at the channel exit flash with the channel walls it is possible to narrow the plasma plume. The plume narrowing is accompanied with an increase of the voltage drop inside the channel. ${ }^{22}$ In the present experiments, the channel narrowing has an opposite effect on the plasma potential distribution. Within accuracy of plume measurements, we measured a $<10 \%$ larger plume angle for the narrow channel as compared to the wide channel (a half plume angle $\sim 48^{\circ}-50^{\circ}$ ). Interesting that as the discharge voltage increases above $600 \mathrm{~V}$, the difference in the plume angle diminishes, but the thruster with narrow channel operates more efficiently (Fig. 12). The channel erosion is more critical for high discharge voltage operation because of a larger energy of ions impinging the channel walls. It would be interesting in the future to conduct plasma measurements at higher discharge voltages than those used in these experiments, to evaluate the channel erosion, and to find a scaling law for the channel width as a function of the discharge voltage.

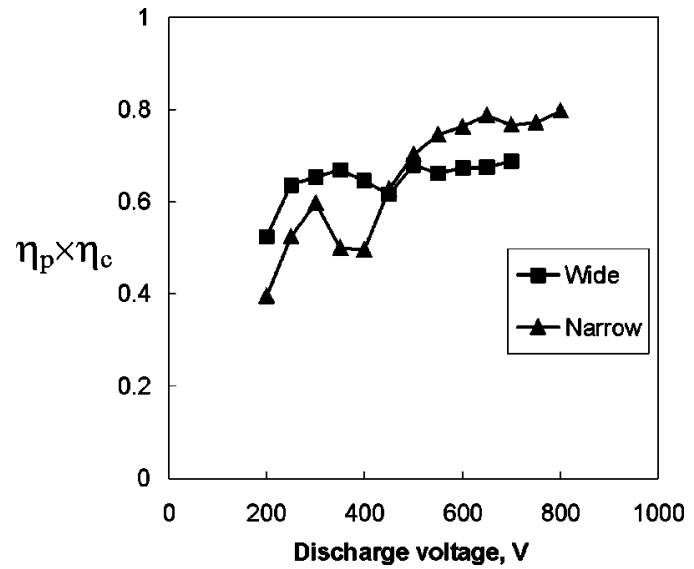

FIG. 12. Propellant utilization times current utilization for xenon gas flow of 19 SCCM and two thruster configurations with two different channel widths: $25 \mathrm{~mm}$ (wide) and $15 \mathrm{~mm}$ (narrow). Magnetic field is not changed.

\section{CONCLUSIONS}

Recent theoretical studies of Hall thrusters predicted that in the presence of strong secondary electron emission, electron-wall collisions can significantly affect electron energy losses on the channel walls and electron crossed-field transport. A characteristic frequency of electron-wall collisions depends on the distance between the channel walls, what we call the "channel width." We have conducted experiments in which the channel width was varied. The discharge voltage was also varied to affect the Joule heating. Using an emissive probe the plasma potential and the electron temperature were measured in the acceleration region of a $2 \mathrm{~kW}$ Hall thruster. The experimental dependence of the maximum electron temperature on the discharge voltage exhibits the discharge voltage threshold $\sim 400 \mathrm{~V}$. Below the voltage threshold, the maximum temperature increases nearly linear with the discharge voltage. The electron energy gain is almost unchanged in the acceleration region inside the channel and in the near-field plasma plume. This result suggests a minor role of the secondary electron emission in electron energy losses on the channel walls. Above the voltage threshold, the maximum temperature saturates. The theory also predicts the temperature saturation in a transition to the space-charge limited regime of the near-wall sheaths. However, the measured saturation temperatures are three times larger than the critical value predicted for space-charge saturated near-wall sheath under the assumption of Maxwellian EDF for electrons.

Perhaps the most unexpected result of this study is that it is the channel width, more than the discharge voltage, which influences the location of the accelerating voltage drop variations. In the discharge voltage range of 200-500 V, the channel narrowing significantly reduces the electric field inside the channel and causes the accelerating voltage drop to be established mainly in the near-field plasma plume. Enhanced anomalous crossed-field mobility (near-wall or Bohm conductivity) is suggested by a hydrodynamic model as an explanation to the reduced electric field measured inside the narrow channel. The measured effect of the channel width on the acceleration region is also much stronger than one could 
expect from the existing theories of near-wall conductivity. It is also not so evident from the measurements that the location of the voltage drop depends on the electron mean energy, which would be the case for the SEE-induced near-wall conductivity. The strongest effect of the channel width was actually measured below the voltage threshold where secondary electron emission effects are minor. On the other hand, it is not obvious why Bohm conductivity would be affected directly by the channel width. Therefore, although our present measurements and modeling do not identify precisely the mechanisms of the electron conductivity enhancement due to channel width narrowing, it is believed that the electron-wall interaction contributes directly or indirectly to these observations. More work has to be done in order to understand these mechanisms and their dependences on the channel width and the magnetic mirrors near the channel walls, including kinetic simulations and measurements.

\section{ACKNOWLEDGMENTS}

The authors are grateful to Artem Smirnov for insightful discussions and help in preparation of this paper. The authors wish to thank also Dr. Leonid Dorf, Dr. Alexander Dunaevsky, Professor Amnon Fruchtman, and Professor Ian Boyd for helpful discussions. This work was supported by U.S. Department of Energy under Contract No. AC02-76CH03073.

${ }^{1}$ A. I. Morozov and V. V. Savel'ev, in Reviews of Plasma Physics, edited by B. B. Kadomtsev and V. D. Shafranov (Consultants Bureau, New York, 2000), Vol. 21, p. 203; A. I. Morozov, Plasma Phys. Rep. 29, 235 (2003). ${ }^{2}$ V. Kim, J. Propul. Power 14, 736 (1998).

${ }^{3}$ A. Fruchtman and N. J. Fisch, Phys. Plasmas 8, 56 (2001).

${ }^{4} \mathrm{~V}$. Hruby, Proceedings of the 39th Joint Propulsion Conference and Exhibit, Huntsville, AL, July 2003 (American Institute of Aeronautics and Astronautics, Reston, VA, 2003), AIAA Paper No. 2003-4441.

${ }^{5}$ D. Manzella, R. Jankovsky, and R. Hofer, Proceedings of the 38th Joint Propulsion Conference and Exhibit, Indianapolis, IN, July 2002(American Institute of Aeronautics and Astronautics, Reston, VA, 2002), AIAA Paper No. 2002-3676.

${ }^{6}$ A. Dunaevsky, Y. Raitses, and N. J. Fisch, Phys. Plasmas 10, 2574 (2003).

${ }^{7}$ M. Keidar, I. Boyd, and I. I. Beilis, Phys. Plasmas 8, 5315 (2001).

${ }^{8} \mathrm{~L}$. Jolivet and J-F. Roussel, Proceedings of the Third International Conference on Spacecraft Propulsion, (ESA Publication Division, Noordwijk, The Netherlands, 2000), p. 367.

${ }^{9}$ S. Barral, K. Makowski, Z. Peradzynski, N. Gascon, and M. Dudeck, Phys. Plasmas 10, 4137 (2003).

${ }^{10}$ E. Ahedo, J. M. Gallardo, and M. Martinez-Sanchez, Phys. Plasmas 10, 3397 (2003); E. Ahedo and D. Escobar, J. Appl. Phys. 96, 983 (2004).

${ }^{11}$ G. D. Hobbs and J. A. Wesson, Plasma Phys. 9, 85 (1967).

${ }^{12}$ L. A. Schwager, Phys. Fluids B 5, 631 (1993).

${ }^{13}$ T. Intrator, M. H. Cho, E. Y. Wang, N. Hershkowitz, D. Diebold, and J. DeKock, J. Appl. Phys. 64, 2927 (1988).

${ }^{14}$ P. C. Stangeby, The Plasma Boundary of Magnetic Fusion Devices, Plasma Physics Series (IOP, Bristol, 2000), pp. 646-654.

${ }^{15}$ N. Gascon, M. Dudeck, and S. Barral, Phys. Plasmas 10, 4123 (2003).
${ }^{16}$ J. Ashkenazy, Y. Raitses, and G. Appelbaum, Phys. Plasmas 5, 2055 (1998).

${ }^{17}$ N. B. Meeazan and M. A. Cappelli, Phys. Rev. E 66, 036401 (2002).

${ }^{18} \mathrm{O}$. Batishchev and M. Martinez-Sanchez, Proceedings of the 28th International Electric Propulsion Conference, Toulouse, France, March 2003, (Electric Rocket Propulsion Society, Cleveland, OH, 2003), IEPC paper 2003-188.

${ }^{19}$ A. Smirnov, Y. Raitses, and N. J. Fisch, Phys. Plasmas 11, 4922 (2004).

${ }^{20}$ K. Sullivan, J. Fox, M. Martinez-Sanchez, and O. Batishchev, Proceedings of the 40th Joint Propulsion Conference and Exhibit, Ft. Lauderdale, FL, July 2004, (American Institute of Aeronautics and Astronautics, Reston, VA, 2004), AIAA Paper No. 2004-3777.

${ }^{21}$ I. Kaganovich, M. Misina, S. V. Berzhnoi, and R. Gijbels, Phys. Rev. E 61, 1875 (2000)

${ }^{22}$ Y. Raitses, M. Keidar, D. Staack, and N. J. Fisch, J. Appl. Phys. 92, 4906 (2002)

${ }^{23}$ Y. Raitses, L. A. Dorf, A. A. Litvak, and N. J. Fisch, J. Appl. Phys. 88, 1263 (2000); N. J. Fisch, Y. Raitses, L. A. Dorf, and A. A. Litvak, ibid. 89, 2040 (2001); A. Fruchtman, N. J. Fisch, and Y. Raitses, Phys. Plasmas 8, 1048 (2001).

${ }^{24}$ Y. Raitses, J. Ashkenazy, and M. Guelman, J. Propul. Power 14, 247 (1998).

${ }^{25}$ Y. Raitses, D. Staack, L. Dorf, and N. J. Fisch, Proceedings of 39th Joint Propulsion Conference and Exhibit, Huntsville, AL, July 2003, (American Institute of Aeronautics and Astronautics, Reston, VA, 2003), AIAA Paper No. 2003-5153.

${ }^{26}$ D. Staack, Y. Raitses, and N. J. Fisch, Rev. Sci. Instrum. 75, 393 (2004).

${ }^{27}$ L. Dorf, Y. Raitses, and N. J. Fisch, Rev. Sci. Instrum. 75, 1255 (2004).

${ }^{28}$ A. Smirnov, Y. Raitses, and N. J. Fisch, J. Appl. Phys. 94, 852 (2003).

${ }^{29}$ R. R. Hofer and R. S. Jankovsky, Proceedings of the 28th International Electric Propulsion Conference, Toulouse, France, March 2003, (Electric Rocket Propulsion Society, Cleveland, OH, 2003), IEPC paper 2003-142.

${ }^{30}$ L. Dorf, Y. Raitses, N. J. Fisch, and V. Semenov, Appl. Phys. Lett. 84, 1070 (2004)

${ }^{31}$ A. Smirnov, Y. Raitses, and N. J. Fisch, J. Appl. Phys. 95, 2283 (2004).

${ }^{32}$ G. Guerrini, A. N. Vesselovzorov, M. Bacal, and I. B. Pokrovsky, Rev. Sci. Instrum. 67, 990 (1996).

${ }^{33}$ J. M. Haas and A. D. Gallimore, Phys. Plasmas 8, 652 (2001).

${ }^{34}$ D. Staack, Y. Raitses, and N. J. Fisch, Appl. Phys. Lett. 84, 3028 (2004).

${ }^{35}$ G. J. M. Hagelaar, J. Bereilles, L. Garrigues, and J.-P. Boeuf, J. Appl. Phys. 93, 67 (2003).

${ }^{36}$ E. Ahedo, J. M. Gallardo, and M. Martinez-Sanchez, Phys. Plasmas 9, 4061 (2003).

${ }^{37}$ M. Keidar, A. D. Gallimore, Y. Raitses, and I. D. Boyd, Appl. Phys. Lett. 85, 2481 (2004).

${ }^{38}$ G. S. Janes and R. S. Lowder, Phys. Fluids 9, 1115 (1966).

${ }^{39}$ N. B. Meezan, W. A. Hargus, and M. A. Cappelli, Phys. Rev. E 63, 026410 (2001).

${ }^{40}$ A. A. Ivanov, A. A. Ivanov, Jr., and M. Bacal, Plasma Phys. Controlled Fusion 44, 1463 (2002).

${ }^{41}$ M. Keidar, I. Beilis, R. L. Boxman, and S. Goldsmith, J. Phys. D 29, 1973 (1996).

${ }^{42}$ A. M. Bishaev and V. Kim, Sov. Phys. Tech. Phys. 23, 1055 (1978).

${ }^{43}$ R. R. Hofer and A. D. Gallimore, Proceedings of 38th Joint Propulsion Conference and Exhibit, Indianapolis, IN, July 2002, (American Institute of Aeronautics and Astronautics, Reston, VA, 2002), AIAA Paper No. 2002-4111.

${ }^{44}$ L. Garrigues G. J. M. Hagelaar, J. Bereilles, C. Boniface, and J.-P. Boeuf, Phys. Plasmas 10, 4886 (2003).

${ }^{45}$ A. Fruchtman and A. Cohen-Zur, Proceedings of the 40th Joint Propulsion Conference and Exhibit, Ft. Lauderdale, FL, July 2004, (American Institute of Aeronautics and Astronautics, Reston, VA, 2004), AIAA Paper No. 2004-3957. 